Document downloaded from:

http://hdl.handle.net/10251/171137

This paper must be cited as:

Conde, P.; González Martínez, AJ.; Hernández, L.; Bellido, P.; Crespo, E.; Iborra, A.; Moliner, L.... (2013). Statistical moments of scintillation light distribution analysis with dSiPMs and monolithic crystals. IEEE. 10-13. https://doi.org/10.1109/NSSMIC.2013.6829086

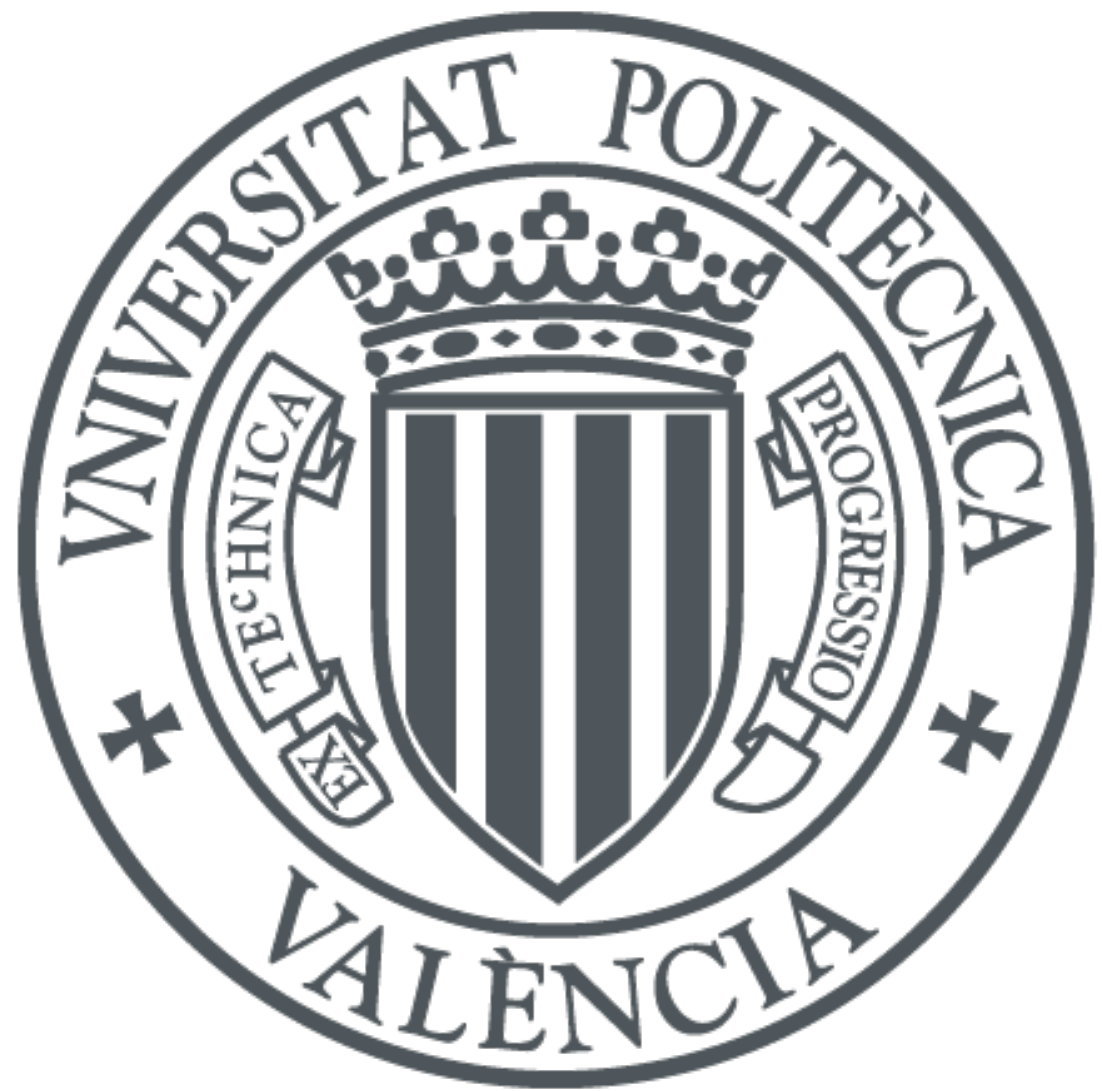

The final publication is available at

https://doi.org/10.1109/NSSMIC.2013.6829086

Copyright IEEE

Additional Information 


\title{
Statistical moments of scintillation light distribution analysis with dSiPMs and monolithic crystals
}

\author{
P. Conde*, A. J. González, L. Hernández, P. Bellido, E. Crespo, A. Iborra, L. Moliner, J. P. Rigla, \\ M. J. Rodríguez-Álvarez, F. Sánchez, M. Seimetz, A. Soriano, L. F. Vidal and J. M. Benlloch.
}

\begin{abstract}
Monolithic scintillation crystals offer the possibility to preserve the scintillation light distribution, specially when black painted. Furthermore, the statistical moments of that distribution can provide accurate information about the three spatial components.

Nevertheless, for monolithic crystal the moments estimation has an associated error due to the symmetry truncation of the light distribution towards the crystal borders. For the 2-D impact coordinates determination, this error is called compression as it is accentuated near the edges. The computation of all centered moments is, therefore, affected by this error.

Digital SiPMs (dSiPMs) can offer complete information about the light distribution, since all cells are purely digital detectors, so that other ways to obtain $\gamma$-impact coordinates can be performed. In this work, a comparison between the statistical moments analysis and an alternative fitting the light distribution for each event to a theoretical distribution has been made. With the fitted approach, compression is avoided and an approximately constant spatial resolution is obtained for the entire photodetection area. Moreover, DOI information is improved and preserved all over the crystal.
\end{abstract}

\section{Materials And Methods}

\section{A. Introduction}

In Positron Emission Tomography (PET), each $511 \mathrm{keV} \gamma$ photon that impacts the scintillator crystal converts its energy into visible light or ultraviolet photons that are isotropically emitted from the excited scintillation centers. In the case of one single elementary interaction, i.e. a photoelectric effect that gives rise to a single photoelectron, the excited scintillation centers are densely accumulated around this photoconversion position, because the range of the electron inside the scintillator will be short. Thus, the distribution of the scintillation light, observed at the plane of the photocathode, will be the same as for a point source located at the same position where the photoconversion took place. This can be expressed by the inverse square law:

$$
J\left(\mathbf{r}, \mathbf{r}_{\mathbf{c}}\right)=\frac{J_{c}}{4 \pi\left|\mathbf{r}-\mathbf{r}_{\mathbf{c}}\right|^{2}}, \quad \mathbf{r} \in \mathbb{R}^{3}
$$

Manuscript received November 15, 2013. This work was supported by the Spanish Plan Nacional de Investigación Científica, Desarrollo e Innovación Tecnológica (I+D+I) under Grant No. FIS2010-21216-CO2-01 and Valencian Local Government under Grants PROMETEOII/2013/010 and ISIC 2011/013

P. Conde, A.J. González, L. Hernández, P. Bellido, E. Crespo, A. Iborra, L. Moliner, J. P. Rigla, M. J. Rodríguez-Álvarez, F. Sánchez, M. Seimetz, A. Soriano, L. F. Vidal and J.M. Benlloch are with the Institute for Instrumentation in Molecular Imaging (I3M), CSIC - Universidad Politécnica de Valencia CIEMAT, Camino de Vera s/n, Ed. 8-N-1 ${ }^{\mathrm{a}}, 46022$ Valencia, Spain.

*Corresponding author. Contact: pabconca@i3m.upv.es. where $J_{c}$ is the number of photons generated at the photoconversion and $\mathbf{r}_{\mathbf{c}}$ the coordinates of the $\gamma$-photon impact.

The projection onto one axis of the inverse square law can be written as:

$$
J\left(x, x_{c}\right)=\frac{J}{\pi} \frac{\sigma_{x}}{\left(x-x_{c}\right)^{2}+\sigma_{x}^{2}}
$$

where $\sigma_{x}=\left(z_{c}-z_{0}\right)$ and $z_{0}$ is the scintillator plane located just over the photosensor detector.

\section{B. 3-D Impact Coordinates determination}

In arrays of SiPM-based detectors, in order to reduce the number of channels to digitized, the impact coordinates can be determined with the first three moments of the sampled distribution, when referring to monolithic crystals [1].

$$
\begin{aligned}
\mu_{n, m} & =\int x^{n} y^{m} J(x, y) d x d y \\
\mu_{n, m}^{\prime}\left(x_{c}, y_{c}\right) & =\int\left(x-x_{c}\right)^{n}\left(y-y_{c}\right)^{m} J(x, y) d x d y
\end{aligned}
$$

Equation (3) shows the two dimensional expression for the statistical moments and equation (4) for the two dimensional centered moments where $x, y$ are the coordinates for each photodetector, i.e. SiPM, and $x_{c}, y_{c}$ the measured coordinates of the center of gravity $(\mathrm{CoG})$. The zeroth moment $\mu_{0}$ corresponds to the total charge released by a $\gamma$-event which is proportional to the detected photon energy. The first moments $\mu_{1,0}$ and $\mu_{0,1}$ are the mean of the light distribution, providing information about the center of gravity $(\mathrm{CoG})$ for each event. The most important centered moment is the variance, $\mu_{2,0}^{\prime}$ and $\mu_{0,2}^{\prime}$, that are a measure for the width of the distribution which are strongly correlated with depth of interaction (DOI) [2]. Note that for symmetric distributions, $\mu_{2,0}^{\prime}=\mu_{0,2}^{\prime}$.

In monolithic crystals the distribution symmetry is truncated everywhere except for the crystal center area, so the moments estimation have an associated error, as depicted in Figure 1. For the first moment, this error is called compression as it is accentuated near the edges. This error in the determination of the centroid affects also the computation of the second centered moment, in the way that the DOI is bad estimated for $\gamma$-rays impacting near the crystal borders. The third centered moment, named skewness, provides information about the asymmetry of a distribution, so it is correlated with the degree of truncation of the sampled light distribution and can be used for further corrections on the $\mathrm{CoG}$ algorithm. 


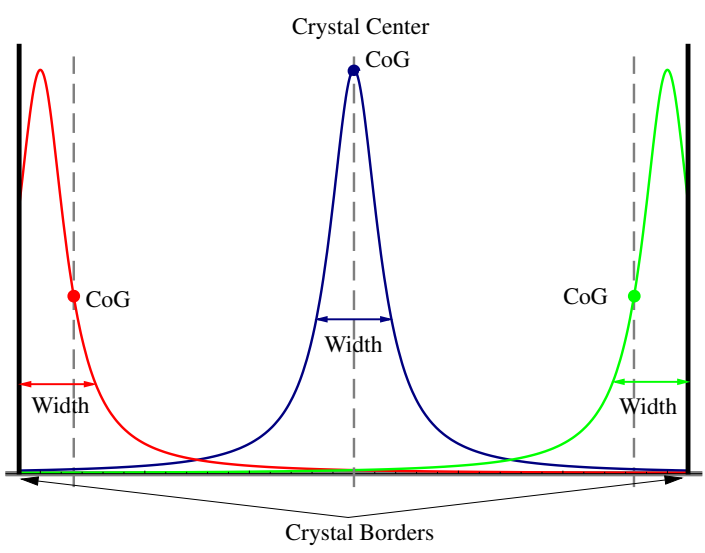

Fig. 1. Projections over one axis of the inverse square law. The truncation of the distribution towards the crystal borders induces errors in the CoG and width estimation.

\section{EXPERIMENTAL SETUP}

Digital SiPMs, manufactured by Philips Digital Photon Counting, consists of 16 independent die sensors, placed on one tile in a $4 \times 4$ matrix covering an area of $32.6 \times 32.6 \mathrm{~mm}^{2}$. Each of these die sensors is further separated into four pixels, arranged in a $2 \times 2$ matrix, so that it has 64 digital outputs. Each of these outputs provides information on the number of photons detected and a correlated timestamp [3].

The acquisition sequence is fully digital, as depicted in Figure 2. All these parameters, i.e. trigger scheme, validation and integration time, can be digitally set [4].

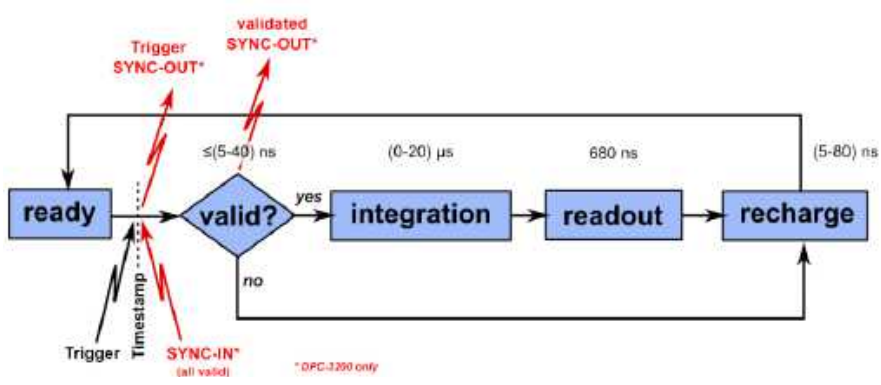

Fig. 2. dSiPM acquisition sequence scheme.

The trigger scheme and validation can be used to determined an energy threshold, reducing the number of dark counts. But as the scintillation light is expected to cover the entire photodetector surface, the dies located far from the scintillation center will not pass such threshold and therefore will not be triggered. To avoid this effect, the neighbor dies can be forcefully triggered to start the acquisition sequence by use of the neighbor logic.

Since all the 3200 cells of each pixel are digital sensors, the information of the sampled distribution from each event is available, thus projections over the planar axis can be fitted to the theoretical model described by the equation (2), where $J$, $x_{c}$ and $\sigma_{x}$ are the fitted parameters. By means of fitting the measured light distributions, the edge compression effect can be avoided and minimized the error associated to the width determination.

As a counterpart of the fitting approach, only photoelectric events can be fitted to the theoretical distribution because in the case that one or more Compton interactions precede the photoelectric interaction that finally absorbs the incident $\gamma$-ray, one has various regions of activated scintillation centers.

All experiments were carried out with two dSiPMs tiles for coincidence measurements, separated by a fixed distance of $10 \mathrm{~cm}$. Both modules were coupled with optical grease to black painted monolithic LYSO crystals of $32.6 \times 32.6 \mathrm{~mm}^{2}$ covering the whole photodetection area. Two crystals heights were used, 10 and $12 \mathrm{~mm}$.

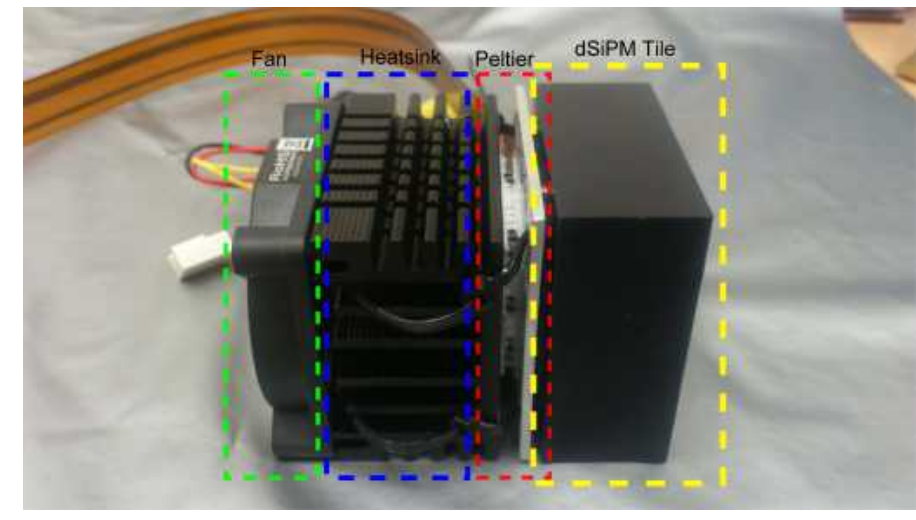

Fig. 3. dSiPM detector module.

In order to reduce the dark count rate a custom made Peltier cooling system was developed with a Peltier module coupled through thermal paste to a heatsink and fan block, so that both modules were kept at a stable temperature of $T=20^{\circ} \mathrm{C}$. A picture of the detector module is shown in Figure 3. An inhibit map (only possible with dSiPMs) deactivating the cells with higher dark counts than $10 \%$ was created.

Since the scintillation distribution of the light is expected to cover the whole photodetection area, the neighbor logic of the dSiPMs tiles was fully activated. The validation time was set to $40 \mathrm{~ns}$, which is mandatory when the neighbor logic is activated, while the integration time was set to $45 \mathrm{~ns}$ which is the approximate decay time for a LYSO crystal. Moreover, due to the high dark count rate at $T=20^{\circ} \mathrm{C}$, the trigger scheme was set to the fourth probabilistic photon. The coincidence time window was set to a relativily high value of $50 \mathrm{~ns}$ due to the timestamp skew between dies of each sensor and the timestamp skew between different tiles, that has not yet calibrated in our setup. A tungsten collimated ${ }^{22} \mathrm{Na}$ source of $1 \mathrm{~mm}^{2}$ in diameter was placed near the detector block with the $10 \mathrm{~mm}$ LYSO crystal and was sequentially moved along the $\mathrm{X}$ axis using a digital controlled stepper motor.

\section{EXPERIMENTAL RESULTS}

\section{A. Energy Resolution}

Summing up the number of detected photons for each pixel that is inside the integration time of $45 \mathrm{~ns}$, the total charge released in the photodetection area is measured. In order to obtain an accurate energy resolution of the detector under 

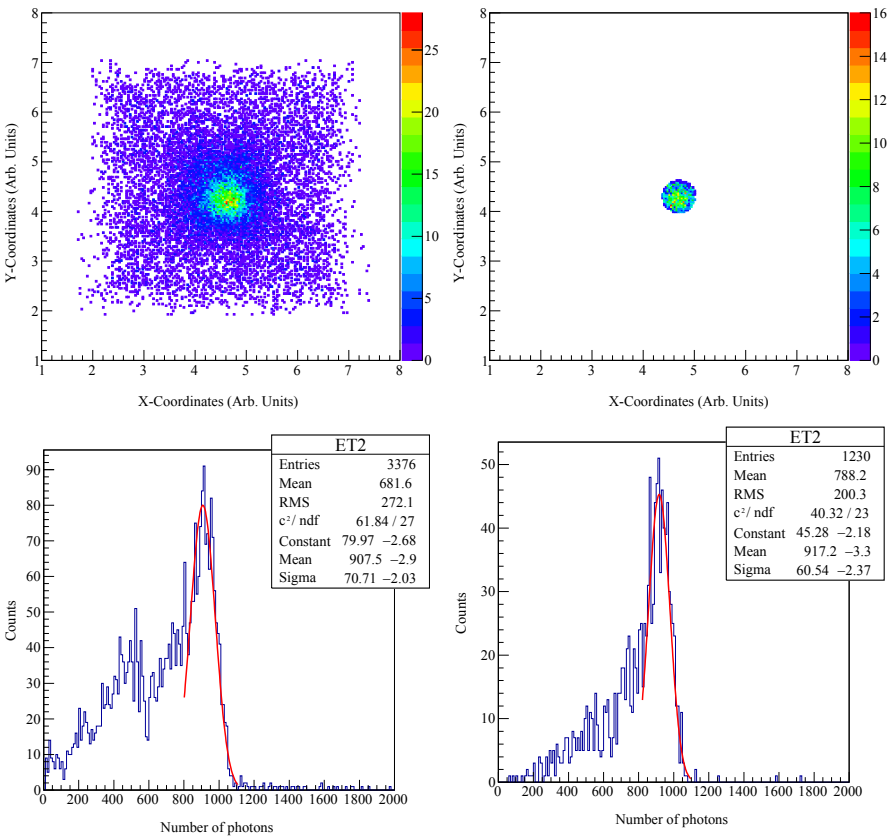

Fig. 4. Left, tungsten collimated source in front of the detector under analysis and electronical collimation on the opposite. Right, additional region of interest collimation.
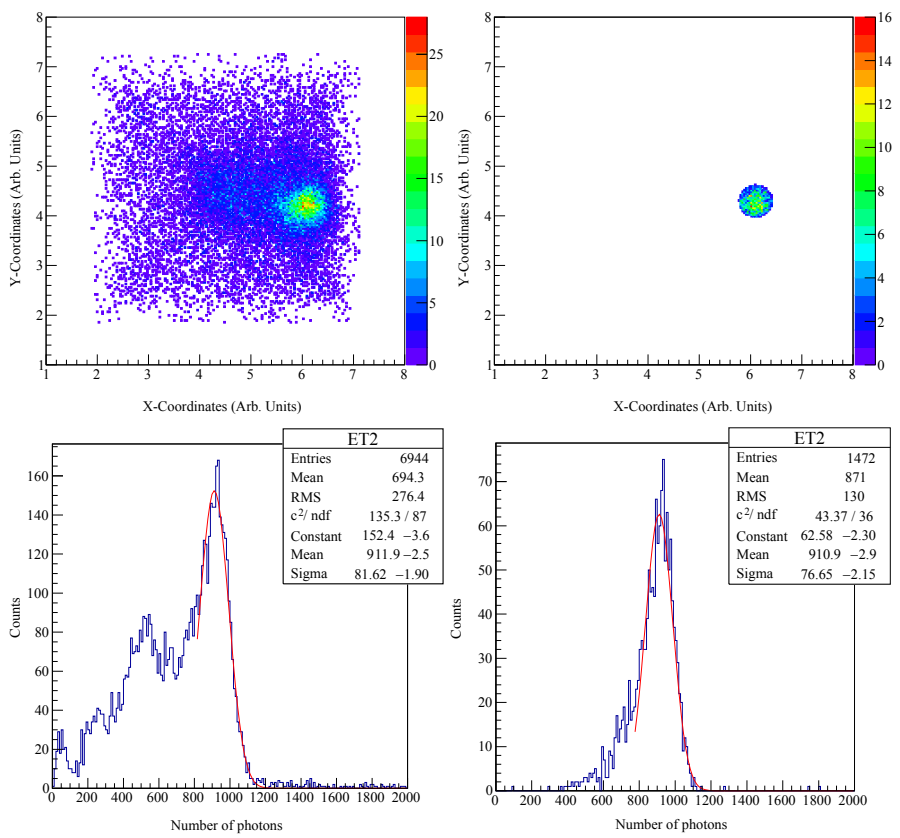

Fig. 5. Left, tungsten collimated source placed near one border of the detector under analysis and electronical collimation on the opposite. Right, additional region of interest collimation.

study, events are electronically collimated, that is selecting a region of interest (ROI) over the coincident detector, and in a $10 \%$ window around the photopeak are taken into account.

An energy resolution of $18.3 \pm 0.1 \%$ and $15.5 \pm 0.1 \%$ was determined, when considering events generated with the ${ }^{22} \mathrm{Na}$ source in the entire crystal volume and for a central ROI, respectively. When the collimated source is placed near the crystal edge, the energy resolution degrades to $21.0 \pm 0.1 \%$ and $19.8 \pm 0.1 \%$, respectively, as can be seen in Figures 4 and 5 .

\section{B. Spatial Resolution}

As is depicted in Figure 6 the linearity between the real impact coordinates and measured coordinates when these are determined using the $\mathrm{CoG}$ algorithm, is lost except for a small region near the crystal center. However, when the 2-D impact coordinates are obtained by fitting the distribution of every single event to the theoretical light distribution the linearity is preserved for the entire photodetection surface.

The spatial resolution obtained using the first moment of the sampled light distribution becomes worse towards the crystal edges due to the truncation of the distribution but, as expected, the spatial resolution obtained with the fitting approach remains constant for the entire crystal surface, as shown in Figure 7.

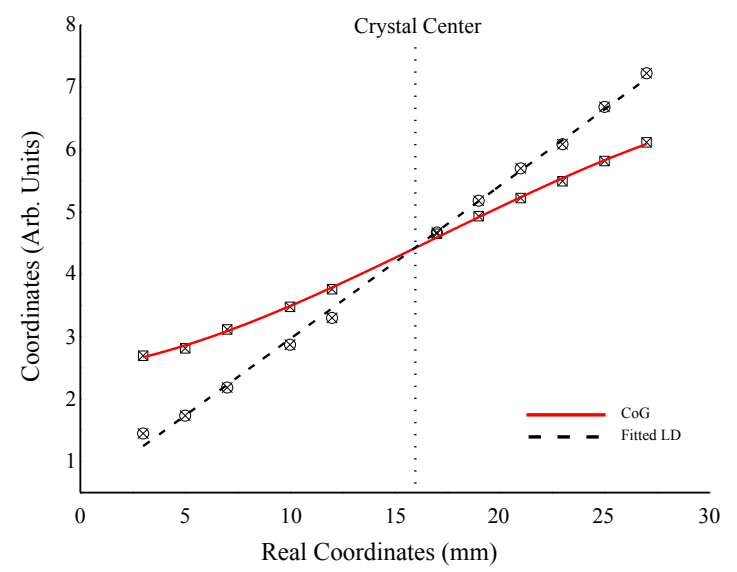

Fig. 6. Plot of measured versus real coordinates for different impact positions, comparing the results provided by the $\mathrm{CoG}$ and fitting approaches.

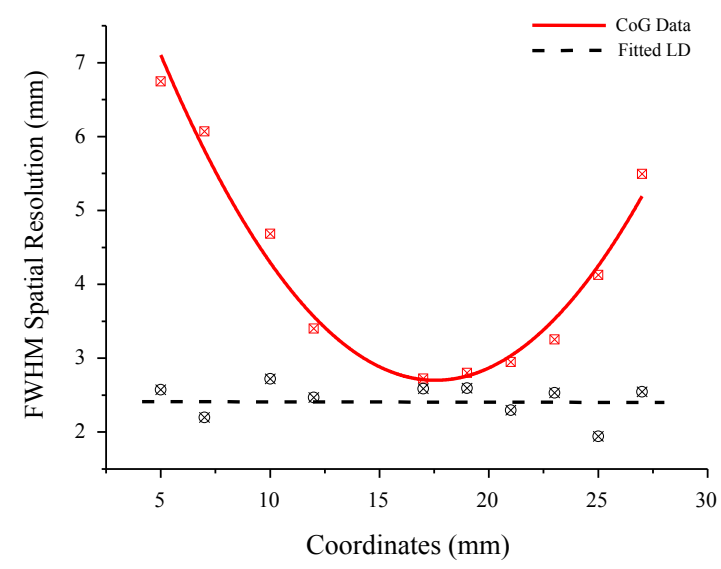

Fig. 7. Plot of results for the spatial resolution along one detector axis for the CoG and light distribution fitting approaches. The solid and dashed lines are, a second and first order polynomial fits to the values, for CoG and Fitted data, respectively.

\section{DOI Resolution}

For a collimated $\gamma$-ray beam impinging normal to the detector, the measured DOIs are randomly distributed, following 
an exponentially decay distribution. Therefore, the distribution of the measured second order moments or the fitted width parameter is expected to be a superposition of the detector intrinsic DOI resolutions at different depths, weighted by a falling exponential due to the $\gamma$-ray attenuation, as shown in Figure 8.

The intrinsic depth resolution $\triangle D O I^{\prime}$ can be related to the crystal thickness $T$ by scaling it with the constant factor:

$$
\Delta D O I(m m)=\Delta D O I^{\prime} \frac{T}{|a-b|}
$$

where $a, b$ are the crystal limits along the $z$-axis.

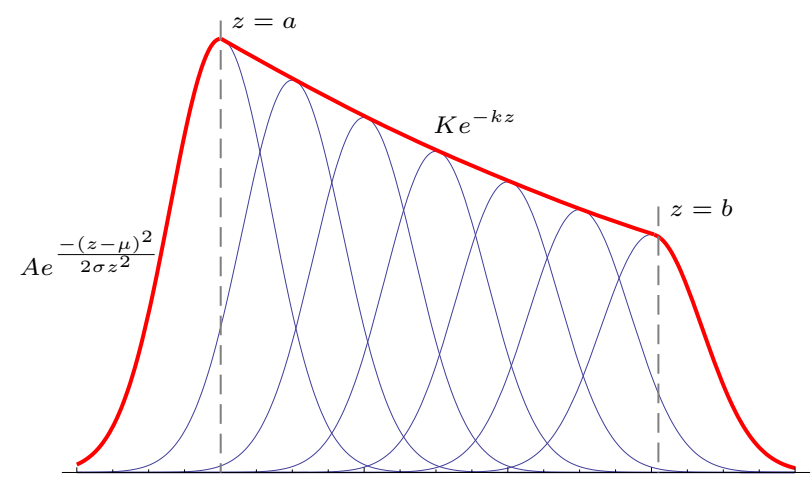

Fig. 8. Theoretical distribution of DOIs for a $\gamma$-ray beam impinging perpendicular to the photodetector array. Parameters $a, b$ represents the crystal limits, and $K e^{-k z}$ is the attenuation function.

In Figure 9 it can be seen that the DOI resolution $(\triangle D O I)$ degrades towards the crystal borders when measured using the second centered moment, as a consequence of the light distribution truncation. At the crystal center $\triangle D O I$ is $3.1 \pm$ $0.1 \mathrm{~mm}$ and at the crystal borders is about $7.1 \pm 0.4 \mathrm{~mm}$. As in the 2-D impact coordinates determination, the DOI resolution obtained using the fitted distributions is not affected at the

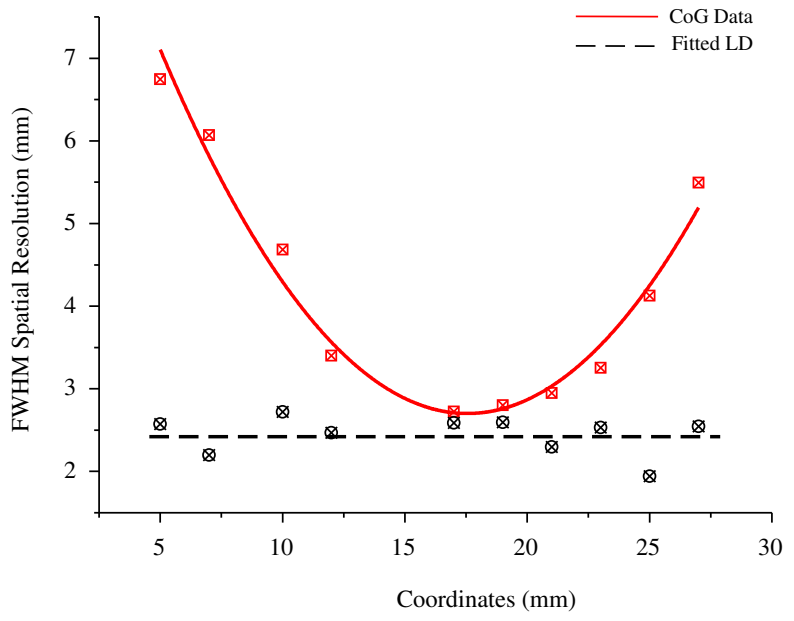

Fig. 9. Comparison between the measured DOI resolutions at different source positions using the second centered moment algorithm and the fitting approach. The solid and dashed lines are a second and first order polynomial fits to the values for $\mathrm{CoG}$ and fitted data, respectively. border and therefore remains approximately constant for the entire crystal surface. Moreover, the width estimation for each event is more accurate following the fitting procedure, so a better DOI resolution of $2.8 \pm 0.1 \mathrm{~mm}$ is measured.

\section{Conclusions And Outlook}

An energy resolution about $18 \%$ is reached, with black painted monolithic crystals at a stable temperature of $T=$ $20^{\circ} \mathrm{C}$. Although not shown in this work, by lowering the temperature of the sensor assembly, the energy resolution can be improved. A spatial resolution (FWHM) about $3 \mathrm{~mm}$ at the crystal center and $7 \mathrm{~mm}$ at the crystal edges has been measured using the $\mathrm{CoG}$ algorithm. Fitting the light distribution of every single event to a theoretical function, most of the border effects can be suppressed, and a global spatial resolution of about 2.5 $\mathrm{mm}$ is measured for the entire crystal area. A DOI resolution about $3.1 \mathrm{~mm}$ was found at the crystal center and $7.4 \mathrm{~mm}$ at the crystal border, using the second centered moment. With the fitted model a DOI resolution about $2.8 \mathrm{~mm}$ was found for the entire crystal.

The timestamp skew between dSiPMs dies, and also between different dSiPM tiles, forces to use broad time windows for coincident measurements worsening the signal to noise ratio and also adding some pile-up effects. Calibration of this skew can further improve the results.

\section{REFERENCES}

[1] P. Conde, A. González, L. Hernández, P. Bellido, A. Iborra, E. Crespo, L. Moliner, J. Rigla, M. Rodríguez-Álvarez, F. Sánchez, M. Seimetz, A. Soriano, L. Vidal, and J. Benlloch, "Results of a combined monolithic crystal and an array of ASICs controlled SiPMs," Nuclear Instruments and Methods in Physics Research Section A: Accelerators, Spectrometers, Detectors and Associated Equipment, no. 0, pp. -, 2013. [Online]. Available: http://www.sciencedirect.com/science/article/pii/S0168900213012254

[2] C. Lerche, A. Ros, R. Gadea, R. Colom, F. Toledo, V. Herrero, J. Monzo, A. Sebastia, D. Abellan, F. Sanchez, C. Correcher, A. Gonzalez, A. Munar, and J. Benlloch, "Doi measurement with monolithic scintillation crystals: A primary performance evaluation," in Nuclear Science Symposium Conference Record, 2007. NSS '07. IEEE, vol. 4, 2007, pp. 2594-2600.

[3] Y. Haemisch, T. Frach, C. Degenhardt, and A. Thon, "Fully digital arrays of silicon photomultipliers (dSiPM) - a scalable alternative to vacuum photomultiplier tubes (pmt)," Physics Procedia, vol. 37, no. 0, pp. 1546 - 1560, 2012.

[4] T. Frach, G. Prescher, C. Degenhardt, R. de Gruyter, A. Schmitz, and R. Ballizany, "The digital silicon photomultiplier; principle of operation and intrinsic detector performance," in Nuclear Science Symposium Conference Record (NSS/MIC), 2009 IEEE, 2009, pp. 1959-1965. 